\title{
Illusory contour perception in domestic dogs
}

\section{Sarah-Elizabeth Byosiere ${ }^{1}$ (D) Philippe A. Chouinard ${ }^{1} \cdot$ Tiffani J. Howell $^{1} \cdot$ Pauleen C. Bennett $^{1}$}

Published online: 4 September 2019

(C) The Psychonomic Society, Inc. 2019

\begin{abstract}
One way to better understand how animals visually perceive their environment is to assess the way in which visual information is interpreted and adapted based on preconceptions. Domestic dogs represent a unique species in which to evaluate visual perception as recent findings suggest they may differ from humans and other animal species in terms of their susceptibility to geometric visual illusions. Dogs have demonstrated human-like, reversed, and null susceptibility depending on the type of illusion. To further evaluate how dogs perceive their environment, it is necessary to perform additional assessments of visual perception. One such assessment is the perceptual filling-in of figures, which may be invoked when presented with illusory contours. Six dogs were assessed on their perception of the Ehrenstein illusory contour illusion in a two-choice size-discrimination task. Dogs, as a group, demonstrated equivocal perception of illusory contours. Some individual dogs, however, demonstrated human-like perception of the subjective contours, providing preliminary evidence that this species is capable of perceiving illusory contour illusions, thereby improving the current understanding of canine visual perception capabilities. Additional assessments using alternative illusory contour illusions are needed to clarify these results and identify features that underpin the individual differences observed.
\end{abstract}

Keywords Dog $\cdot$ Ehrenstein $\cdot$ Illusion $\cdot$ Perception $\cdot$ Illusory contour

\section{Introduction}

Visual perception represents an inventive process. Not only does the brain receive information from a visual scene from the retina, but it also interprets this information and adapts it to fit preconceptions (Gregory, 2015). Therefore, the perception of a visual scene may not accurately reflect reality but instead the most likely scene based on an individual's previous experiences (Kandel \& Schwartz, 2000). It is in these instances that misperceptions can occur: When mechanisms that are generally helpful for accurately perceiving the environment trick the brain into applying preconceptions to visual information to represent the most likely visual scene.

Illusory contours represent one such type of misperception. These are experienced when individuals recognise and mentally complete figures or shapes that are missing connective contours (Kanizsa, 1974). The process makes use of available

Sarah-Elizabeth Byosiere

sebyosiere@gmail.com

1 School of Psychology and Public Health, La Trobe University, P.O. Box 199, Bendigo, Victoria 3552, Australia information such as foreground/background brightness, color, and textures, so that when an object in its entirety is incomplete, the missing components are imagined. One example of an illusory contour, initially described by Schumann (1900), is the Ehrenstein illusion (Ehrenstein, 1987) (see later in Methods). This illusion involves amodal completion, also known as the perceptual filling of gaps in a visual scene. In other words, the viewer mentally percieves boundaries that are physically not present.

To date, illusory contour perception has been assessed in a variety of non-human animals (hereafter referred to as animals) (Bravo, Blake, \& Morrison, 1988 Fagot \& Tomonaga, 2001 Fuss, Bleckmann, \& Schluessel, 2014 Kanizsa, Renzi, Conte, Compostela, \& Guerani, 1993 Nieder \& Wagner, 1999 Sovrano \& Bisazza, 2009 van Hateren, Srinivasan, \& Wait, 1990 Wyzisk \& Neumeyer, 2007; Zimmermann, 1962; Zylinski, Darmaillacq, \& Shashar, 2012) to improve our understanding of how they perceive the world and to determine similarities and/or differences between humans. The majority of studies have done so by evaluating perception of Kanizsa figures. Chimpanzees (Fagot \& Tomonaga, 2001), rhesus macaques (Zimmermann, 1962), cats (Bravo et al., 1988), mice (Kanizsa et al., 1993), barn owls (Nieder \& Wagner, 1999), 
bamboo sharks (Fuss et al., 2014), redtail splitfin fish (Sovrano \& Bisazza, 2009), and goldfish (Wyzisk \& Neumeyer, 2007) have all indicated human-like misperception of illusory contours in Kanizsa figures. Taken together, these findings suggest that there may be similarities in the development of visual processing and the shared and/or nonshared neural mechanisms regarding illusory contour perception (Banica \& Schwarzkopf, 2016).

While many species demonstrate human-like susceptibility to geometric visual illusions (Feng, Chouinard, Howell, \& Bennett, 2017), one species in particular, the domestic dog, has demonstrated variable findings (Byosiere et al., 2017; Byosiere et al., 2016; Byosiere et al., 2018; Keep, Zulch, \& Wilkinson, 2018; Miletto Petrazzini, Bisazza, \& Agrillo, 2016). Depending on the illusion (i.e., EbbinghausTitchener, Delboeuf, and Ponzo illusions), dogs have demonstrated reversed or null susceptibility compared to humans and many other non-human animals (Byosiere, Feng, Rutter, et al., 2017; Byosiere et al., 2016; Byosiere et al., 2018; Miletto Petrazzini et al., 2016). In humans, susceptibility across different illusions likely represents multiple independent mechanisms rather than a single construct invoked by the same cognitive processes (Chouinard, Noulty, Sperandio, \& Landry, 2013; Chouinard, Unwin, Landry, \& Sperandio, 2016), which may explain the variable findings observed in dogs. Nonetheless, the extent of this deviation warrants additional explanation.

Given their unique relationship with humans and the everincreasing rise of canine cognition research in the last two decades (Bensky, Gosling, \& Sinn, 2013), it is instructive to better understand how dogs perceive their external environment. An improved understanding of their perception may increase the efficacy of research methodologies and training methods, which ultimately affect the dog-human relationship. Therefore, the current research aimed to evaluate the perception of illusory contours in domestic dogs, specifically perception of the Ehrenstein illusory contour illusion.

\section{Methods}

The sample initially consisted of nine purebred Lagotto Romagnolo dogs (Canis familiaris) (Table 1). Six subjects successfully completed discrimination training and participated in the test experiment. Three subjects did not move on to the test experiment as they were not able to successfully complete the discrimination trainings. It is important to note that as a part of a larger on-going project, many of the dogs that participated in this experiment underwent an ophthalmological examination (Byosiere et al., 2018) and a test of visual acuity (Graham et al., 2018). These assessments revealed no abnormalities such as myopia or hypermetropia (i.e., nearsightedness or farsightedness) that may have affected visual
Table 1 Subject demographics at the time of testing

\begin{tabular}{lll}
\hline Name & Sex & Age (years) \\
\hline Audrey & Female & 2.3 \\
Baxter $^{*}$ & Male & 3 \\
Beth $^{*}$ & Female & 5.5 \\
Cricket $^{*}$ & Female & 10 months \\
Eliza $^{*}$ & Female & 4 \\
Gabbie $^{1}$ & Female & 3.6 \\
Hamish $^{\text {Lulu }}$ & Male & 7.8 \\
Molly & Female & 4 \\
\hline
\end{tabular}

${ }^{1}$ Subject desexed

* Subject trained to select smaller of two presented stimuli

acuity. All procedures were approved in accordance with $\mathrm{La}$ Trobe University's Animal Ethics Committee (AEC17-14).

\section{Training and testing apparatus}

As a part of a longer-term investigation evaluating visual perception in dogs (Byosiere, Chouinard, Howell, \& Bennett, 2019; Byosiere, Feng, Chouinard, Howell, \& Bennett, 2017; Byosiere, Feng, Rutter, et al., 2017; Byosiere et al., 2016; Graham et al., 2018), subjects were first target-trained to nose-touch a single black circle stimulus. The targeting behavior was then transferred to a two-choice discrimination task in which circles of different sizes were presented in pairs. The majority of dogs were trained to target the larger circle, except for Baxter and Cricket, who were trained to select the smaller circle. Dogs as a group demonstrated high accuracy in discriminating circle sizes of $60 \%$ difference in size, performing at $99 \pm 2.6 \%$ (for an extensive review of the discrimination capabilities of these subjects, see Byosiere et al., 2018).

Testing was conducted in the Canine Nose-Touch Testing Apparatus (Byosiere, Feng, Chouinard, et al., 2017; Byosiere, Feng, Rutter, et al., 2017; Byosiere et al., 2016; Byosiere et al., 2018), which was used to minimize potential experimenter cues and distractions (Fig. 1). The apparatus was equipped with a $508-\mathrm{mm}$ Dell ${ }^{\circledR}$ widescreen computer monitor $(1,280$ $\times 800$ pixels; one pixel measures approximately $0.3 \mathrm{~mm}^{2}$ ) positioned with the lower edge approximately $24 \mathrm{~cm}$ above ground level. At ground level, directly below, was a remotely controlled treat dispenser (Treat \& Train $\left.{ }^{\circledR}\right)$, which when activated, distributed one piece of semi-moist dry dog food (Nature's Gift $₫$ Mini Kangaroo, or Good-o $₫$ treats). A video camera was mounted above the monitor to record each trial. Microsoft PowerPoint was used to create and present stimuli, and data were collected via analyzing and coding videos after testing. A more detailed description of the apparatus is provided by Byosiere et al. (2016). 

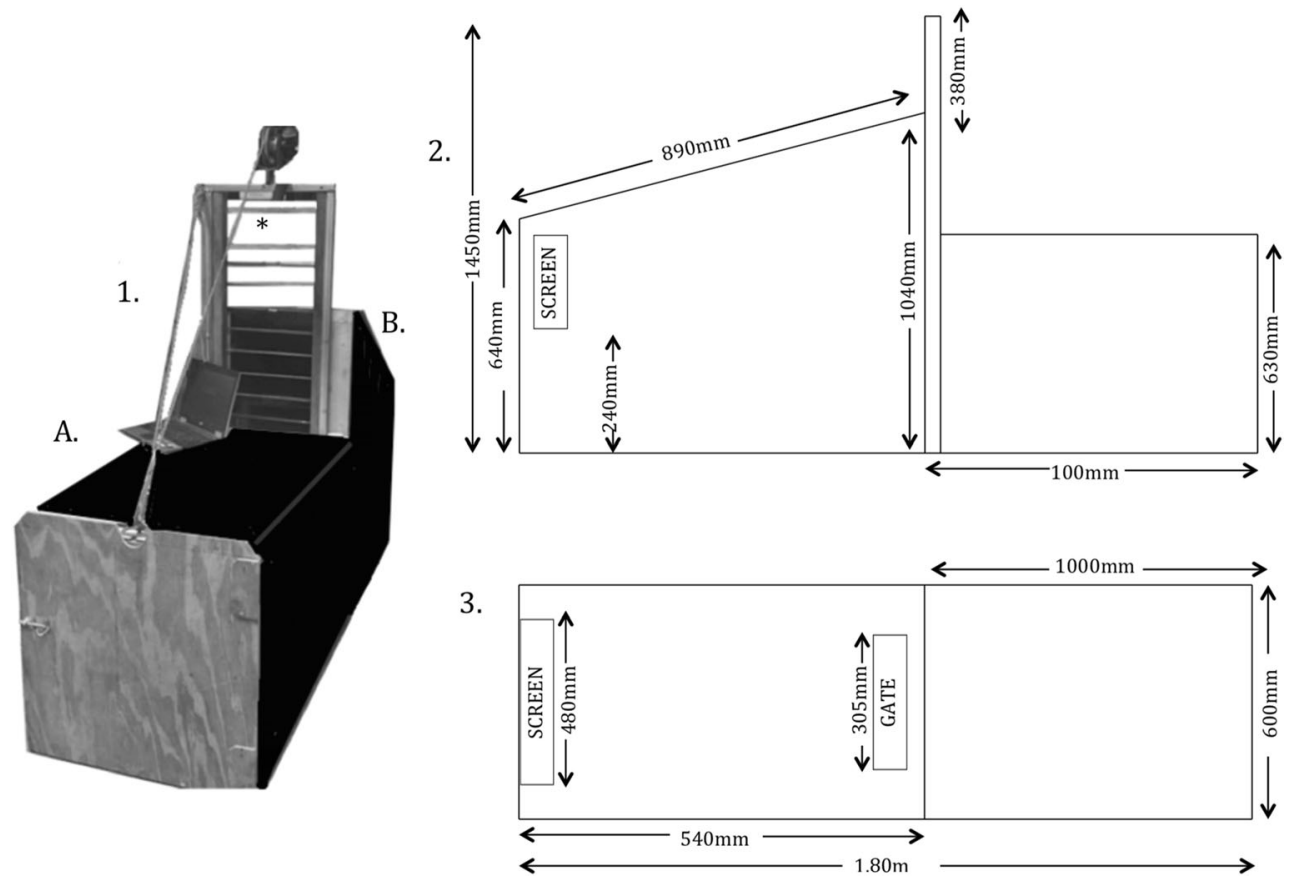

Fig. 1 Photographic representation of the testing apparatus, the Canine Nose-Touch Testing Apparatus, used during training and testing phases (1) with schematic side (2) and aerial (3) diagrams. The figure presents the

positions of Experimenters A and B during the testing process and the asterisk represents the location of the camera

\section{Stimuli}

All stimuli were presented in black (RGB values: $0,0,0$; luminance: $0.18 \mathrm{c} / \mathrm{m}^{2}$ ) and white (RGB values: 255,255 , 255; luminance: $175.5 \mathrm{c} / \mathrm{m}^{2}$ ) on an LCD screen. The target stimuli used were based on those previously used to evaluate illusion susceptibility, size-sensitivity thresholds, and various discrimination tasks in this same group of dogs (Byosiere, Feng, Rutter, et al., 2017; Byosiere et al., 2016; Byosiere et al., 2018). The small circle stimuli were 207 pixels in diameter $(62.1 \mathrm{~mm})$, while the large stimulus was 403 pixels in diameter $(120.9 \mathrm{~mm})$. All lines presented (including the outline of the circle in the Ring Control, Overall Size Control, and Ray Length Control) were 10 pixels $(3 \mathrm{~mm})$ wide. In the Overall Size Control, the lines protruding from the smaller-sized circle were 250 pixels $(75 \mathrm{~mm})$ long, while the lines protruding from the larger-sized circle stimulus were 50 pixels $(15 \mathrm{~mm})$ long. In the Ray Length Control, the lines protruding from the smaller-sized circle were 50 pixels $(15 \mathrm{~mm})$ long, while the lines protruding from the larger-sized circle stimulus were 150 pixels $(45 \mathrm{~mm})$ long. In the Test where subjects were presented with the Ehrenstein illusion, the lines protruding from the smaller-sized circle were 196 pixels $(58.8 \mathrm{~mm})$ long, while the lines protruding from the larger-sized circle stimulus were 98 (29.4) pixels long. Test stimuli were equal in overall size, presenting an image of 599 pixels in diameter $(179.7 \mathrm{~mm})$.

\section{Procedure}

One experimenter was present at all times during testing, out of sight from the dog. The experimenter sat to the left of the Canine Nose-Touch Testing Apparatus; this experimenter controlled a laptop computer (presenting and removing the stimuli) and activated the remote-controlled treat dispenser. Once in the apparatus and unable to see the experimenter, the dog moved to the screen and selected one stimulus (presented on either the left or the right) by nose-touching the image.

Before participating in test trials, each dog completed the necessary discrimination training to ensure they were able to identify the correct target. Dogs were presented with blocks of ten trials. The first trial began when the stimuli were presented on the screen and the dog was positioned at the entrance of the apparatus. All dogs participated in discrimination training and progressed only after reaching a criterion of $90 \%$ or more correct in a single ten-trial block, or $80 \%$ or more correct across two consecutive blocks. To advance to the testing session, each dog was required to complete all discrimination training phases successfully. These phases consisted of blocked presentations of ten total trials, where the first two trials always consisted of a previously seen stimulus (carried over from a previous discrimination training session). Each block took approximately 2 min to complete and dogs participated in five to ten blocks per day. The location of the rewarded stimulus and stimuli presented were randomized 
using a Gellerman sequence (1933). During discrimination training, if the dog chose correctly, the remote-controlled treat dispenser was activated and delivered a food reward. If the dog chose incorrectly, the stimuli were removed, thereby ending the trial. The dog then returned to the entrance of the apparatus to resume the starting position for the next trial. Subjects were excluded from continuing after failing to successfully pass the criteria after a maximum of seven block attempts.

Discrimination Training 1 consisted of two presentations of a size-discrimination task of solid circles and was followed by eight presentations of the Ring Control (Table 2). Discrimination Training 2 and 3 also consisted of two presentations of a size-discrimination task of solid circles but were followed by eight presentations of a ring-like stimulus with lines or rays protruding from it. To ensure the dogs were selecting based on ring size and not overall figure size, the rays protruding from the smaller circle in Discrimination
Training 2 resulted in an overall larger figure, Overall Size Control. In Discrimination Training 3, this was not the case, Ray Length Control.

In the test, dogs participated in ten blocks of ten trials. The first two trials were always the familiar Ring Control presented in Discrimination Training 1. The order of the following eight trials was randomly determined. Two trials presenting the stimuli from Discrimination Training 2 (Overall Size Control) and Discrimination Training 3 (Ray Length Control) were conducted. Four presentations of the Ehrenstein illusory contour illusion were presented. Therefore, each subject completed 100 trials, 20 trials for each previously seen stimulus, and 40 trials for the illusion. So as not to reinforce any particular way of responding, reinforcement for the experimental trials that evaluated illusory contour perception occurred randomly as dictated by a randomly selected Gellermann (1933) sequence. In other words, if the Gellerman sequence indicated that the circle on

Table 2 Stimuli presented in discrimination trainings 1, 2, 3, and the test

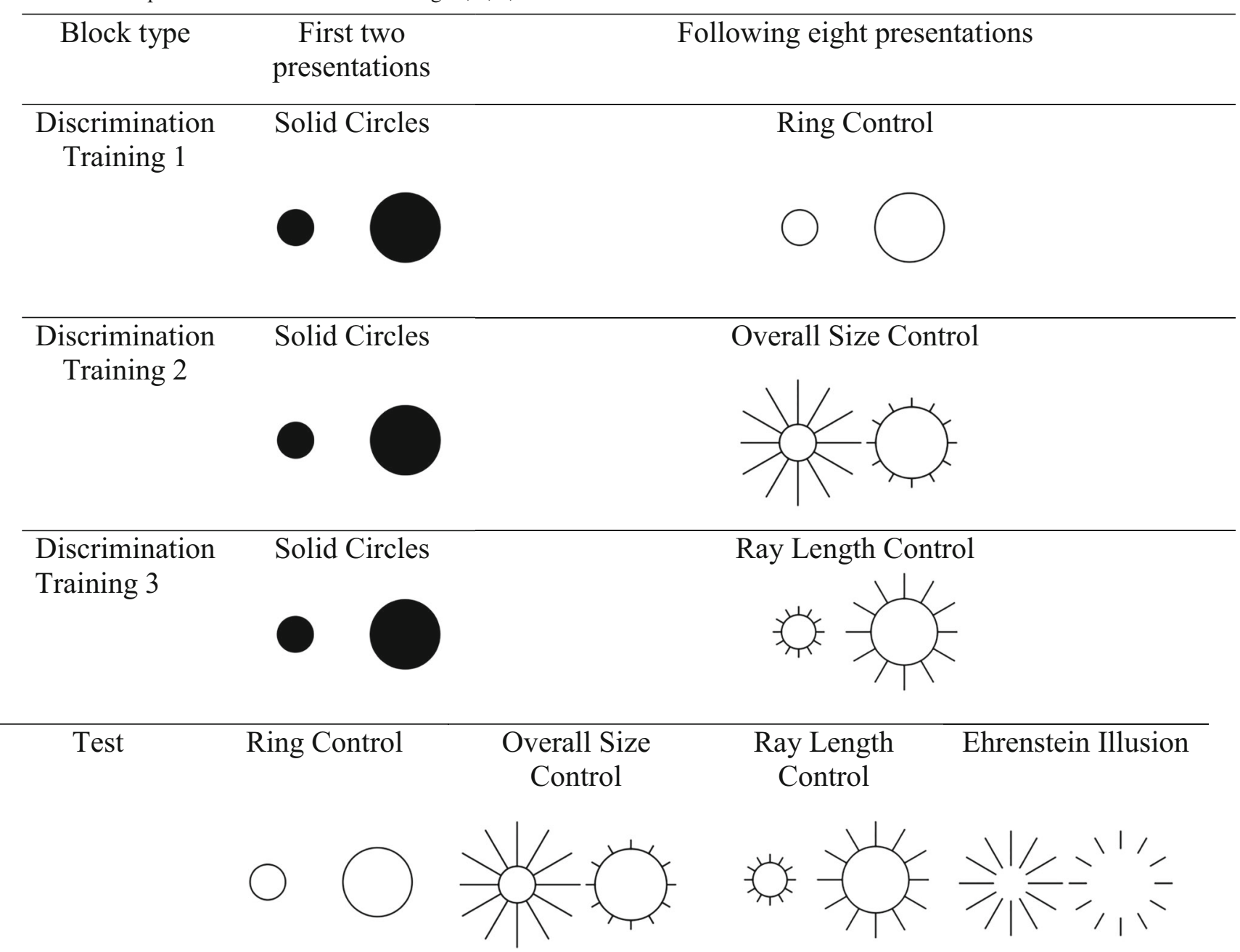

Dogs trained to select the larger circle were expected to select the stimulus on the right, whereas dogs trained to select the smaller circle were expected to select the stimulus on the left 
the left for a particular test trial should be correct, it was deemed thus for the purposes of reinforcement. This was the case even though the circles were identical in size and even though the alternate circle may have been "correct" in so far as humans perceived it as perceptually larger. Once a dog made a choice, the stimuli were removed from the screen, and the reward was delivered.

\section{Data analysis}

Descriptive statistics for the discrimination trainings were conducted to ensure passing criteria were met before each experiment. To determine if dogs were performing significantly above chance, two-tailed non-parametric binomial tests were conducted at both the individual and the group level to examine the discrimination performance of the various stimulus presentations. In addition to null hypothesis statistical testing, Bayes factors were calculated at the group level for the control and illusion conditions to quantify evidence in support for either the null or the alternative hypothesis relative to the other (Wetzels et al., 2011). Bayes factors $\left(\mathrm{BF}_{10}\right)$ denote the likelihood of the alternative over the null hypothesis. Therefore, a $\mathrm{BF}_{10}$ value greater than 3 was considered to provide substantial support for the alternative hypothesis, while a value below .333 was considered to provide substantial support for the null. To evaluate if there were any differences between the stimulus presentations at the level of the group, a non-parametric Friedman test of differences among repeated measures was conducted. In the case of a significant Friedman result, Dunn's tests for multiple comparisons were conducted at the level of the group. Alpha was set at 0.05 and chance levels were considered to be 0.5 , barring the Dunn's test, in which a Bonferonni correction for multiple comparisons was applied and significance was set at 0.0125 .

\section{Results}

Nine dogs participated at the onset of Experiment 1; however, only six subjects successfully passed through all discrimination trainings. All nine dogs successfully completed Discrimination Training 1 in the first block. In Discrimination Training 2, the nine dogs averaged 4.56 blocks (ranging from 1 to 7) before successfully meeting criteria in order to continue on to Discrimination Training 3. During this phase, three dogs (Beth, Eliza, and Hamish) were unable to meet passing criteria within seven total blocks and were excluded from continuing in the experiment. In Discrimination Training 3, the six remaining dogs averaged two blocks (ranging from 1 to 5 ) before successfully moving on to the test.

Figure 2 shows individual and group performance for each dog in the test sessions. To determine whether the dogs' proportion of correct choices differed significantly from chance
(.50), two-tailed binomial tests were conducted. Performance when presented with the Ring Control was high, indicating that the proportion of correct choices, 95\% \pm 5.5 (standard deviation), for the group was significantly higher than expected chance performance $\left(p<0.001\right.$, Cohen's $d=8.21, \mathrm{BF}_{10}=$ 2347.71). When presented with the stimulus presented in Discrimination Training 2, where the rays of the smaller ring extended beyond that of the larger ring (Overall Size Control), group performance was $77.5 \% \pm 14.4$ and was significantly higher than expected by chance ( $p<0.001$, Cohen's $d=1.91$, $\left.\mathrm{BF}_{10}=10.92\right)$. When presented with the stimulus presented in Discrimination Training 3, where the rays of the larger ring extended beyond that of the smaller ring (Ray Control), performance was significantly above chance at $83.3 \% \pm 8.2(p<$ 0.001 , Cohen's $\left.d=4.08, \mathrm{BF}_{10}=158.02\right)$. Finally, when presented with the Ehrenstein illusory contour illusion, percent correct (defined by human-like perception) as a group decreased and variability (in terms of standard deviation) increased, with the dogs averaging $59.2 \% \pm 21.1$. As a group, null hypothesis statistical testing revealed that the proportion of correct choices was significantly higher than expected by chance ( $p=0.005$, Cohen's $d=0.44)$. However, a Bayes factor failed to fully support either the null or the alternative hypothesis $\left(\mathrm{BF}_{10}=.572\right)$, although greater support may be drawn for the null given this value was below 1 .

Individually, variation in performance across the stimulus presentations was observed. All dogs performed significantly above chance in the Ring Control Condition. Molly and Lulu performed at chance in the Overall Size Control Condition. In the Ray Length Control condition, Lulu performed at chance levels. When presented with the Ehrenstein illusion, Baxter and Cricket performed significantly above chance levels, indicating human-like perception of the illusion. Audrey, Gabbie, and Lulu did not perform significantly above chance. Finally, Molly performed significantly below chance, a result opposite to expectation. It is important to note that generally the dog's performance remained consistent across the ten blocks. Audrey, Baxter, Cricket, Gabbie, and Lulu individually differed no more than $5 \%$ in the Ehrenstein illusion in their first five blocks, compared to their last five blocks (Molly differed by 15\%).

To determine whether performance differed across the stimulus presentations, a non-parametric Friedman's test was conducted. All possible comparisons were made, which rendered a significant finding $\left(\chi^{2}(4)=13.07, p<0.01\right)$. Upon further analysis of Dunn's test for multiple comparisons, a significant difference (with a Bonferonni correction of $\alpha<$ 0.0125 ) was found between the Ring Controls and Ehrenstein illusory contour illusion ( $p=0.002)$. No significant differences between the Ring Controls, Overall Size Controls, and Ray Length Controls were observed $(p>0.34)$. No significant differences were observed between the Overall Size 


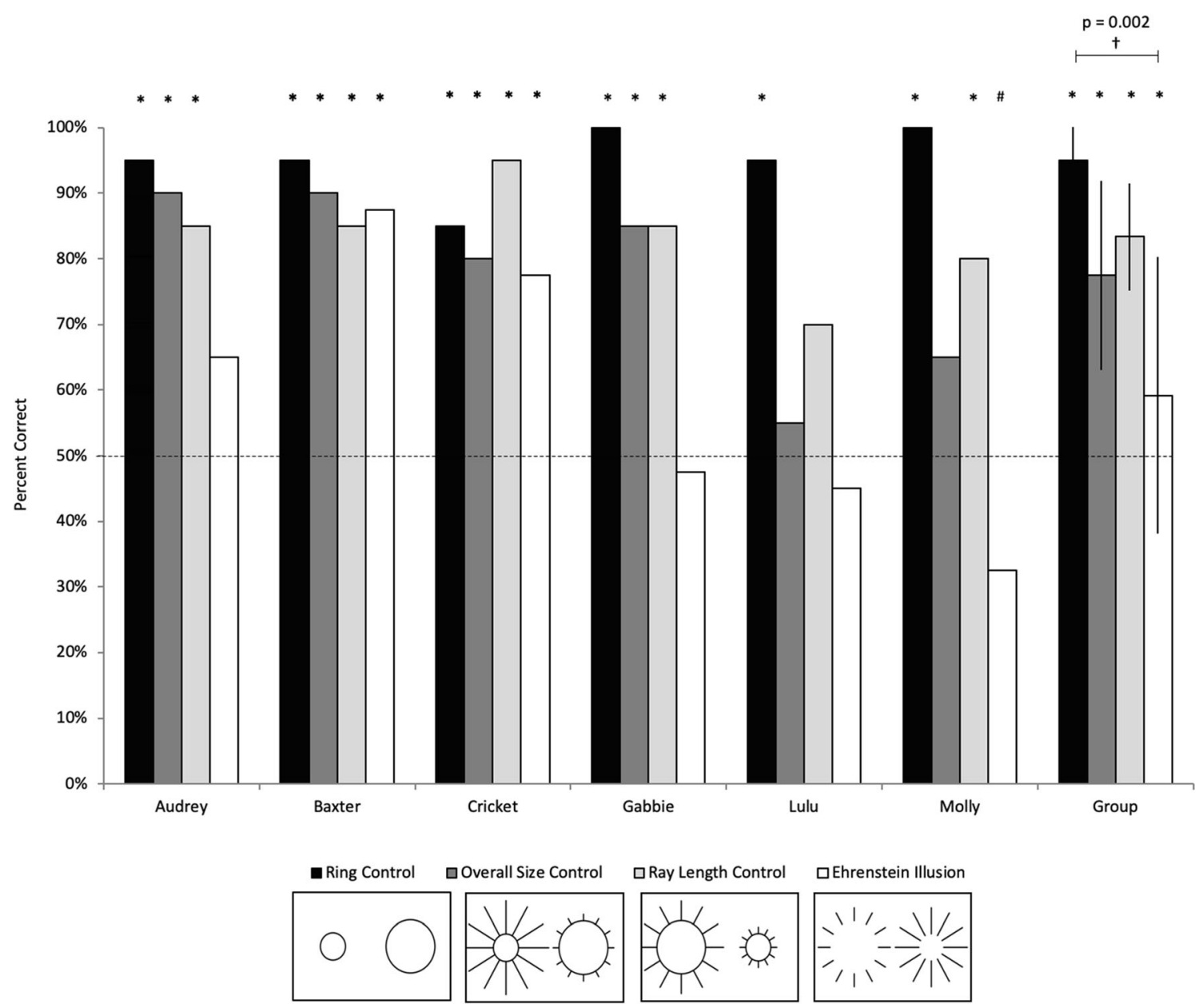

Fig. 2 Individual and group percent correct (and standard deviations indicated as error bars) for control and illusion conditions. * Indicates that the binomial result significantly differed from chance performance demonstrating human-like perception. \# Indicates that the binomial result significantly differed from chance performance demonstrating reversed perception (compared to humans). $\dagger$ Indicates a significant difference in group performance between conditions
Controls, Ray Length Controls, and the Ehrenstein illusory contour illusion $(p>0.6)$.

\section{Discussion}

To assess whether or not dogs demonstrated perception of illusory contours, we evaluated canine perception of the Ehrenstein illusory contour illusion. Evidence for humanlike perception was weak and equivocal. Performance as a group was low, with dogs selecting the illusory figure at only $59.2 \%$. While null hypothesis testing revealed this result was significantly different from chance performance, additional statistics in the form of a Bayes factor failed to provide support for either the null or alternative hypothesis (although greater support could be garnered for the null hypothesis). In comparing group performance across stimulus presentations, results suggested that the dogs were not equally proficient at discriminating between the stimuli. Performance in the illusion condition differed significantly from performance in the Ring Control. No significant difference in performance was observed between the illusion condition and either the Overall Size or the Ray Length Controls. Taken together, these findings provide little evidence that these dogs, as a group, perceive illusory contours.

Individually, there was some evidence to suggest that certain dogs (Baxter, Cricket, and possibly Audrey) demonstrated perception of the illusory figure. One subject, Gabbie, demonstrated null susceptibility, performing at chance when presented with the illusory figures. While these four subjects (Audrey, Baxter, Cricket, and Gabbie) performed with high accuracy on their control trials, other dogs were less successful, and clearly selected stimuli in the test trials based on alternative criteria such as overall size and ray length (Molly), and randomly (Lulu). It is important to note that Lulu and Molly's data must be interpreted cautiously as it is difficult to determine whether or not their lack of susceptibility in the test was reflective of their perception of the illusion or 
an alternative confound (as indicated by chance performance on certain controls). Interestingly, by excluding Molly and Lulu from the analysis, group performance increases to $69.4 \%$ in the illusion condition. At the individual level these findings, at least, provide proof of concept for illusory contour perception in domestic dogs, which must be substantiated by future research.

While the group findings are equivocal, the perception of subjective contours by some individuals is consistent with those observed in other animal species, as well as in humans, and provides preliminary information in regard to canine illusory contour perception. Specifically, these findings suggest that dogs, like humans and many other animals, may experience a separation between what is projected on the retina and what the brain processes (Gregory, 2015; Kandel \& Schwartz, 2000). This would indicate that the canine brain applies existing underlying preconceptions to contexts in which they are not necessary. It is unlikely that contour-detecting cells in the primary visual cortex solely facilitate this perception, as edge detection can be characterized as a complex and multidimensional process in which various cells analyze and extrapolate information from the visual field across the stage(s) of visual processing (Hubel \& Wiesel, 1962). Therefore, it appears that the perception of illusory contours represents the creation of an imaginative figure, produced by a visual system with a proclivity to complete figural components (Gerbino \& Salmaso, 1987; Kanizsa, 1974; Purghé \& Coren, 1992).

\section{Methodological considerations of the present investigation}

Any conclusions drawn from this study are preliminary and additional investigation is required. This is due to the inherent difficulties of verifying that decisions are made by dogs, or any other non-human animal species, on the basis of seeing contours rather than adopting other strategies. These limitations are not unique to animal perception research. In humans, the development of illusory contour perception is highly contested (Nayar, Franchak, Adolph, \& Kiorpes, 2015). While object recognition is generally considered to be a hierarchical process, progressing from elemental and local function to more complex integrative global processing with age, a number of studies provide conflicting reports (for a review, see Nayar et al., 2015). In a study of 4-month-old infants, Freeseman, Colombo, and Coldren (1993) observed that global processing is evident before local processing. Given such wide-ranging controversies, further testing, using additional control conditions and other illusory contour illusions, is required to clarify these preliminary observations in dogs.

We reason that alternative strategies are less likely to underlie the results we obtained, given that these alternative strategies may often require more cognitive analytical reasoning than basing decisions on perceiving illusory contours. The dogs that participated in this experiment had all undergone similar, if not identical, training and testing across a variety of size discrimination, stimulus generalization, and illusion susceptibility tasks (Byosiere et al., 2019; Byosiere, Feng, Chouinard, et al., 2017; Byosiere, Feng, Rutter, et al., 2017; Byosiere et al., 2016; Byosiere et al., 2018; Graham et al., 2018). Moreover, to control for specific factors, all dogs tested were purebred Lagotto Romagnolo dogs that were genetically related, with similar living conditions. Morphological consistency was maintained as morphological variations may affect brain structure and visual processing (McGreevy, Grassi, \& Harman, 2003; Roberts, McGreevy, \& Valenzuela, 2010). This variation does highlight the need to study individual differences within dogs in addition to breed and environmental effects. Such research, within genetically and environmentally similar or dissimilar dogs, could enhance our understanding of dog perception and cognition.

Finally, as these dogs have participated in multiple studies of illusion susceptibility, it is particularly interesting to note that this variation at the individual level is not unique (Byosiere, Feng, Rutter, et al., 2017; Byosiere et al., 2016; Byosiere et al., 2018). Moreover, the human literature has proposed that processing style should not be considered to be a singular construct (Chouinard et al., 2013; Chouinard et al., 2016), but instead should be identified as an umbrella term in which various independent mechanisms are involved in cognitive operations. Given that illusions vary in type and modality, it is not unexpected that different underlying mechanisms and perceptual and cognitive operations are involved. Therefore, multiple assessments of different types of illusions, and even of the same illusion, should be conducted in order to obtain a complete representation of susceptibility at both the individual and the group level.

\section{Conclusion}

It has previously been observed that immense variation exists across geometric visual illusion susceptibility in domestic dogs. Dogs have demonstrated human-like, reversed, and null-illusion susceptibility (Byosiere, Feng, Rutter, et al., 2017; Byosiere et al., 2016; Byosiere et al., 2018; Keep et al., 2018; Miletto Petrazzini et al., 2016). While this may be due to variation in the mechanisms underlying illusory perception, the variation and deviation in susceptibility from other animal species are particularly interesting. To date, perception of illusory contours has not been studied in dogs. Therefore, we evaluated canine perception of the Ehrenstein illusory contour illusion. Equivocal findings were observed regarding the dogs' perception as a group. While nullhypothesis testing revealed evidence for the perception of subjective contours, a finding consistent with those observed 
in other animal species as well as humans, performance was close to chance, and Bayes factors failed to provide support for either the null or the alternative hypothesis. Individually, some dogs did demonstrate perception of the subjective contours of the Ehrenstein illusory contour illusion. These findings provide further proof of concept for the conjecture that, in dogs, additional processing occurs in the brain after visual information enters the retina. However, additional research is needed to substantiate these claims and rule out alternative accounts more definitively.

Acknowledgements We are grateful to Lynna Feng, Nicholas Rutter, and Jessica Woodhead for their help in previous training processes. We also thank Ron Wheeler for his assistance in the construction and design of our apparatus, and Davis Vorva for his expertise in program design. This study was carried out with the support of a La Trobe University Postgraduate Research Scholarship and a La Trobe University Full Fee Research Scholarship.

Open practices statement The data and programs for this experiment are available upon request.

\section{References}

Banica, T., \& Schwarzkopf, D. S. (2016). Induction of Kanizsa contours requires awareness of the inducing context. PLoS One, 11(8), e0161177. https://doi.org/10.1371/journal.pone.0161177

Bensky, M. K., Gosling, S. D., \& Sinn, D. L. (2013). The world from a dog's point of view: A review and synthesis of dog cognition research. Advances in the Study of Behaviour, 45, 209-406. https://doi. org/10.1016/B978-0-12-407186-5.00005-7

Bravo, M., Blake, R., \& Morrison, S. (1988). Cats see subjective contours. Vision Research (28), 861-865. https://doi.org/10.1016/00426989(88)90095-8

Byosiere, S.-E., Chouinard, P. A., Howell, T. J., \& Bennett, P. C. (2019). The effects of physical luminance on colour discrimination in dogs: A cautionary tale. Applied Animal Behaviour Science, 212, 58-65. https://doi.org/10.1016/j.applanim.2019.01.004

Byosiere, S.-E., Feng, L. C., Chouinard, P. A., Howell, T. J., \& Bennett, P. C. (2017). Relational concept learning in domestic dogs: Performance on a two-choice size discrimination task generalises to novel stimuli. Behavioural Processes https://doi.org/10.1016/j. beproc.2017.10.009

Byosiere, S.-E., Feng, L. C., Rutter, N. J., Woodhead, J. K., Chouinard, P. A., Howell, T. J., \& Bennett, P. C. (2017). Do dogs see the Ponzo illusion? Animal Behavior and Cognition. 10.26451/abc/ 04.04.01.2017

Byosiere, S.-E., Feng, L. C., Woodhead, J. K., Rutter, N. J., Chouinard, P. A., Howell, T. J., \& Bennett, P. C. (2016). Visual perception in domestic dogs: susceptibility to the Ebbinghaus-Titchener and Delboeuf illusions. Animal Cognition, 1-14. https://doi.org/10. 1007/s10071-016-1067-1

Byosiere, S.-E., Feng, L. C., Wuister, J., Chouinard, P. A., Howell, T. J., \& Bennett, P. C. (2018). Do dogs demonstrate susceptibility to a vertically presented Ponzo illusion. Animal Behavior and Cognition, 5(3), 254-267.

Chouinard, P. A., Noulty, W. A., Sperandio, I., \& Landry, O. (2013). Global processing during the Müller-Lyer illusion is distinctively affected by the degree of autistic traits in the typical population. Experimental Brain Research, 230(2), 219-231. https://doi.org/10. 1007/s00221-013-3646-6
Chouinard, P. A., Unwin, K. L., Landry, O., \& Sperandio, I. (2016). Susceptibility to optical illusions varies as a function of the autism-spectrum quotient but not in ways predicted by localglobal biases. Journal of Autism and Developmental Disorders, 46(6), 2224-2239. https://doi.org/10.1007/s10803-016-2753-1

Ehrenstein, W. (1987). Modifications of the brightness phenomenon of L. Hermann. In S. Petry \& G. E. Meyer (Eds.), The perception of illusory contours (pp. 35-39). New York, NY: Springer New York.

Fagot, J., \& Tomonaga, M. (2001). Effects of element separation on perceptual grouping by humans (Homo sapiens) and chimpanzees (Pan troglodytes): Perception of Kanizsa illusory figures. Animal Cognition, 4(3), 171-177. https://doi.org/10.1007/s100710100109

Feng, L. C., Chouinard, P. A., Howell, T. J., \& Bennett, P. C. (2017). Why do animals differ in their susceptibility to geometrical illusions? Psychonomic Bulletin \& Review, 24(2), 262-276. https://doi.org/ 10.3758/s13423-016-1133-3

Freeseman, L. J., Colombo, J., \& Coldren, J. T. (1993). Individual differences in infant visual attention: Four-month-olds' discrimination and generalization of global and local stimulus properties. Child Development, 64(4), 1191-1203.

Fuss, T., Bleckmann, H., \& Schluessel, V. (2014). The brain creates illusions not just for us: Sharks (Chiloscyllium griseum) can "see the magic" as well. Frontiers in Neural Circuits, 8, 24. https://doi. org/10.3389/fncir.2014.00024

Gellermann, L. W. (1933). Chance orders of alternating stimuli in visual discrimination experiments. The Pedagogical Seminary and Journal of Genetic Psychology, 42(1), 206-208. https://doi.org/10. 1080/08856559.1933

Gerbino, W., \& Salmaso, D. (1987). The effect of amodal completion on visual matching. Acta Psychologica, 65(1), 25-46. https://doi.org/ 10.1016/0001-6918(87)90045-X

Graham, K. L., Byosiere, S.-E., Feng, L. C., Sanders, M., Bennett, P. C., Caruso, K., . . . White, A. (2018). A forced-choice preferential looking task for the assessment of vision in dogs: pilot study. https://doi.org/10.1111/jsap.12965

Gregory, R. L. (2015). Eye and brain: The psychology of seeing: Princeton university press, Princeton.

Hubel, D. H., \& Wiesel, T. N. (1962). Receptive fields, binocular interaction and functional architecture in the cat's visual cortex. The Journal of Physiology, 160(1), 106. https://doi.org/10.1113/ jphysiol.1962.sp006837

Kandel, E., \& Schwartz, J. (2000). Jessell. TM. Principles of Neural Science. In: New York, NY: McGraw Hill.

Kanizsa, G. (1974). Contours without gradients or cognitive contours? Italian Journal of Psychology, 1(1), 93-112.

Kanizsa, G., Renzi, P., Conte, S., Compostela, C., \& Guerani, L. (1993). Amodal completion in mouse vision. Perception, 22(6), 713-721. https://doi.org/10.1068/p220713

Keep, B., Zulch, H. E., \& Wilkinson, A. (2018). Truth is in the eye of the beholder: Perception of the Müller-Lyer illusion in dogs. Learning \& Behavior, 46(4), 501-512. https://doi.org/10.3758/s13420-0180344-z

McGreevy, P., Grassi, T. D., \& Harman, A. M. (2003). A strong correlation exists between the distribution of retinal ganglion cells and nose length in the dog. Brain, Behavior and Evolution, 63(1), 13-22. https://doi.org/10.1159/000073756

Miletto Petrazzini, M. E., Bisazza, A., \& Agrillo, C. (2016). Do domestic dogs (Canis lupus familiaris) perceive the Delboeuf illusion? Animal Cognition, 1-8. https://doi.org/10.1007/s10071-016-1066-2

Nayar, K., Franchak, J., Adolph, K., \& Kiorpes, L. (2015). From local to global processing: The development of illusory contour perception. Journal of Experimental Child Psychology, 131, 38-55. https://doi. org/10.1016/j.jecp.2014.11.001

Nieder, A., \& Wagner, H. (1999). Perception and neuronal coding of subjective contours in the owl. Nature Neuroscience, 2, 660. https://doi.org/10.1038/10217 
Purghé, F., \& Coren, S. (1992). Subjective contours 1900-1990: Research trends and bibliography. Perception \& Psychophysics, 51(3), 291-304. https://doi.org/10.3758/bf03212255

Roberts, T., McGreevy, P., \& Valenzuela, M. (2010). Human induced rotation and reorganization of the brain of domestic dogs. PLoS One, 5(7), e11946.

Schumann, F. (1900). Beiträge zur Analyse der Gesichtswahrnehmungen. Erste Abhandlung. Einige Beobachtungen über die Zusammenfassung von Gesichtseindruecken zu Einheiten. Zeitschrift für Psychologie und Physiologie der Sinnesorgan (23), $1-32$.

Sovrano, V. A., \& Bisazza, A. (2009). Perception of subjective contours in fish. Perception, 38(4), 579-590. https://doi.org/10.1068/p6121

van Hateren, J. H., Srinivasan, M. V., \& Wait, P. B. (1990). Pattern recognition in bees: Orientation discrimination. Journal of Comparative Physiology A, 167(5), 649-654. https://doi.org/10. 1007/BF00192658

Wetzels, R., Matzke, D., Lee, M. D., Rouder, J. N., Iverson, G. J., \& Wagenmakers, E.-J. (2011). Statistical evidence in experimental
psychology:An empirical comparison using 855 t tests. Perspectives on Psychological Science, 6(3), 291-298. https://doi. org/10.1177/1745691611406923

Wyzisk, K., \& Neumeyer, C. (2007). Perception of illusory surfaces and contours in goldfish. Visual Neuroscience, 24(3), 291-298. https:// doi.org/10.1017/S095252380707023X

Zimmermann, R. R. (1962). Form generalization in the infant monkey. Journal of Comparative and Physiological Psychology, 55(6), 918923. https://doi.org/10.1037/h0042446

Zylinski, S., Darmaillacq, A.-S., \& Shashar, N. (2012). Visual interpolation for contour completion by the European cuttlefish (Sepia officinalis) and its use in dynamic camouflage. Proceedings of the Royal Society B: Biological Sciences, 279(1737), 2386.

Publisher's note Springer Nature remains neutral with regard to jurisdictional claims in published maps and institutional affiliations. 